\title{
SOIL MOISTURE ESTIMATION USING A MULTI-ANGULAR MODIFIED THREE COMPONENT POLARIMETRIC DECOMPOSITION
}

\author{
Thomas Jagdhuber ${ }^{1,2}$, Irena Hajnsek ${ }^{1}$, Konstantinos P. Papathanassiou ${ }^{1}$, Axel Bronstert ${ }^{2}$ \\ ${ }^{1}$ German Aerospace Center \\ Microwaves and Radar Institute, PO BOX 1116, 82234 Wessling \\ ${ }^{2}$ University of Potsdam \\ Institute of Geoecology, Karl-Liebknecht-Strasse 24-25, 14476 Potsdam \\ Email: thomas.jagdhuber@dlr.de, irena.hajnsek@dlr.de,kostas.papathanassiou@dlr.de, axelbron@uni-potsdam.de
}

\begin{abstract}
In this paper a modified three component polarimetric decomposition incorporating multi-angular acquisitions is developed to estimate soil moisture under vegetation cover over agricultural areas. The approach is applied on fully-polarimetric L-band data acquired by DLR's airborne E-SAR sensor in the frame of the OPAQUE campaign conducted in May 2008 in the Weißeritz catchment area, near Dresden, Germany. The results for the estimated soil moisture from the overlapping area of the flight strips demonstrate a significant increase of the inversion rate, if more than one acquisition is used. The inverted soil moisture values are validated against in situ measurements for five test fields with different crop types resulting in an RMSE of approximately $7 \mathrm{vol} . \%$ for different incidence angle constellations. Finally the results show how topographic effects in the soil moisture retrieval can be compensated by multi-angular constellations.
\end{abstract}

Index Terms - Polarimetric decomposition, multiangular, soil moisture

\section{INTRODUCTION}

The capability to use model-based polarimetric decompositions for soil moisture (mv) estimation under vegetation has been already demonstrated for agricultural areas in $[1,2]$. However the results stated that modelbased decompositions are limited to a certain range of local incidence angles $\left(\sim 20^{\circ}\right.$ to $\left.\sim 70^{\circ}\right)[2]$.

Hence exceeding this local angle of incidence (AOI) range the mv inversion leads to non-physical results and no $\mathrm{mv}$ inversion can be performed. In order to enlarge the amount of invertible pixels, one solution is to increase the range of possible AOI by using additional acquisitions.

In this paper the AOI diversity is investigated with regards to the inversion rate of the mv retrieval and the $\mathrm{mv}$ inversion performance by means of the root mean square error (RMSE) between in situ and estimated soil moistures.

\section{POLARIMETRIC DECOMPOSITION USING MULTI-ANGULAR AQUISITIONS}

A modified three component polarimetric decomposition is developed and combined with an eigenvalue decomposition to invert soil moisture under vegetated and bare soils at L-band [1], [2]. The three modified model components of the polarimetric decomposition are surface scattering described with the X-Bragg model, dihedral scattering modeled with a double Fresnel reflection incorporating a vegetation attenuation factor and volume scattering specified as a single volume layer of random or oriented dipoles. This approach is expanded to obtain a modified three component polarimetric decomposition using multi-angular acquisitions for $\mathrm{mv}$ inversion as shown in Fig. 1.

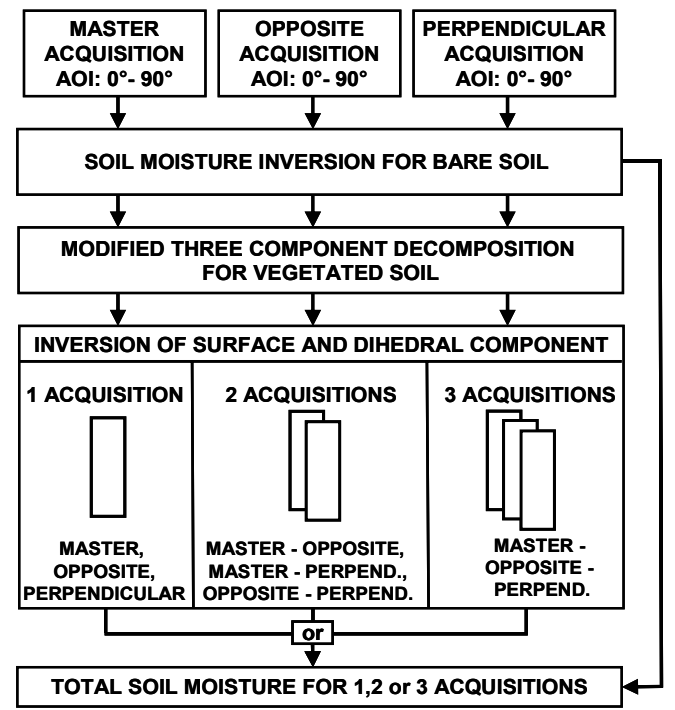

Fig. 1. Processing scheme of the modified three component polarimetric decomposition incorporating multi-angular observations for $\mathrm{mv}$ inversion (perpend.=perpendicular)

The data processing has been done in different steps described in the following. First the three different incidence angle acquisitions (master, opposite, perpendicular) are processed separately for the retrieval 
of soil moisture via the X-Bragg model for bare soil surfaces, which are determined by an $\mathrm{H} / \alpha$-criterion [2]. In a second step the modified three component polarimetric decomposition of [2] is applied to all scenes separately, in which the following changes are included:

- The roughness term of the surface component $(\psi)$ is retrieved from the following relation [3]:

$$
\begin{aligned}
\gamma_{R R L L} & =\frac{\left\langle S_{R R} \cdot S_{L L}^{*}\right\rangle}{\sqrt{\left\langle\left|S_{R R}\right|^{2}\right\rangle \cdot\left\langle\left|S_{L L}\right|^{2}\right\rangle}} \\
\psi & =\pi / 2 \cdot\left(1-\left|\gamma_{R R L L}\right|\right)
\end{aligned}
$$

where $\gamma_{R R L L}$ represents the polarimetric circular coherence.

- The presence of a low vegetation layer $(<25 \mathrm{~cm})$ on the fields in spring, which are still considered as bare soil fields, can bias the soil moisture result. Therefore the surface component of the polarimetric decomposition is also calculated for the pixels, which are previously classified in the first step as bare surfaces.

- The roughness estimation under the vegetation cannot be clearly separated from the vegetation. Hence the dihedral component is only calculated with the vegetation attenuation term $\left(L_{V}\right)$ and without the roughness loss term $\left(L_{S}\right)$.

After the decomposition step the volume $\left(f_{v}\right)$ and ground scattering components (surface: $f_{s}, \beta$, dihedral: $f_{d}, \alpha$ ) are separated. For the inversion of soil moisture from the surface and the dihedral component the parameters $\beta$ as well as $f_{d}$ and $\alpha$ are used. Therefore one, two or three sets of $\alpha, \beta$ and $f_{d}$ can be utilized in an inversion with one, two or three acquisitions, where $k$ is the number of acquisitions. This leads to a maximum of seven different incidence angle constellations. The inversion of $\mathrm{mv}$ in the single acquisition $(k=1)$ case is already demonstrated [2]. For the multi-angular cases $(k=2,3)$ the inversion is carried out as follows:

- For inversion of the surface component the dielectric constant of the soil $\varepsilon_{s}$ is retrieved via a minimization procedure using the sum of absolute differences from the different acquisition cases $k$ between the modeled values $m$ and the data values $d$,

$$
\mathcal{E}_{s}=\min \left(\sum_{n=1}^{k}\left|\beta_{n}^{m}\left(\theta_{n}^{d}, \mathcal{\varepsilon}_{s}\right)-\beta_{n}^{d}\right|\right)
$$

where $\beta^{m}$ denotes the modeled look-up table values with a variety of dielectric constants $\varepsilon_{s}$ and the respective incidence angle $\theta^{d}$ of the data. $\beta^{d}$ is the value from the decomposition of the data.

- For the inversion of the dihedral component the dielectric constant of the soil $\varepsilon_{s}$ has to be inverted together with the dielectric constant of the trunk $\varepsilon_{t}$ due to the dependencies within the Fresnel coefficients. Therefore $\alpha$ and $f_{d}$ are utilized in a combined approach to retrieve $\varepsilon_{s}$ and $\varepsilon_{t}$ :

$$
\varepsilon_{s}, \varepsilon_{t}=\min \left(\begin{array}{l}
\sum_{n=1}^{k}\left|f d_{n}^{m}\left(\theta_{n}^{d}, \varepsilon_{s}, \varepsilon_{t}\right)-f d_{n}^{d}\right| \\
\sum_{n=1}^{k}\left|\alpha_{n}^{m}\left(\theta_{n}^{d}, \varepsilon_{s}, \varepsilon_{t}\right)-\alpha_{n}^{d}\right|
\end{array}\right)
$$

where $f d^{m}$ and $\alpha^{m}$ represent the modeled look-up table values with a variety of $\varepsilon_{s}, \varepsilon_{t}$ as well as the respective incidence angle $\theta^{d}$ of the data. $f d^{d}$ and $\alpha^{d}$ represent the values from the decomposition of the data.

- Finally the dielectric constant of the soil $\varepsilon_{s}$ from both ground components is converted into soil moisture by a universal polynomial transformation [4].

- As a last step the estimated soil moistures from the vegetated and the bare surfaces are unified in one total mv result. For pixels where more than one result from the different methods is present, the average is taken as the final pixel value.

\section{EXPERIMENTAL DATA AND RESULTS}

The algorithm was applied on the data set of the OPAQUE campaign conducted in May 2008 in the Weißeritz catchment area near Dresden, Germany. In the frame of this campaign fully-polarimetric SAR data at Lband were acquired by DLR's airborne E-SAR sensor. Simultaneously, soil moisture and vegetation parameters were measured on selected test fields covering different vegetation and soil types. In Fig. 2 the three multi-angular acquisitions are combined in an RGB composite and are labeled as "master" (m), "opposite" (o) and "perpendicular" (p).

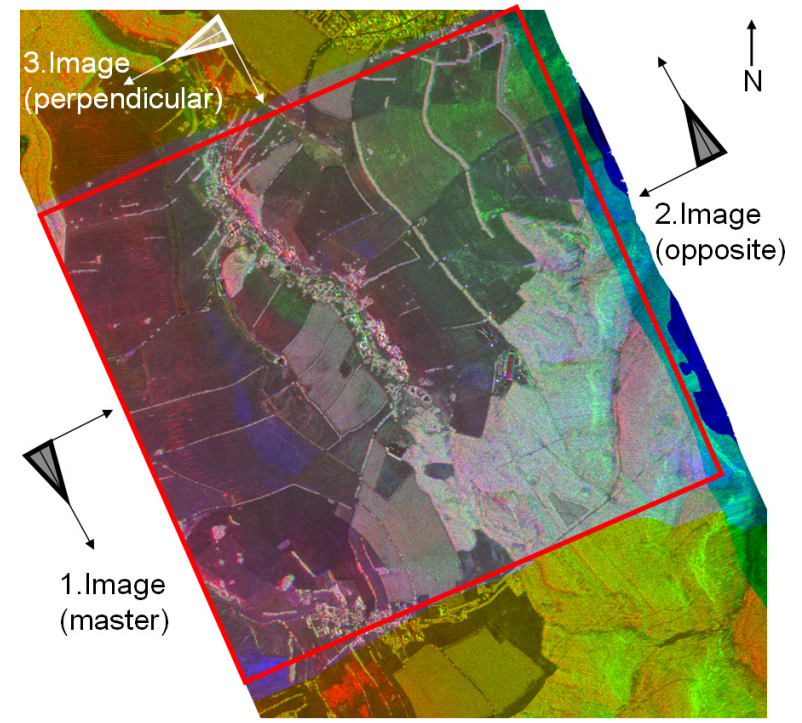

Fig. 2. RGB-composite of the three different incidence angle acquisitions in HH-polarization for L-Band (R: master, G: opposite, B: perpendicular); red frame indicates overlapping area of the three flight strips.

Tab. 1 shows the different AOI of the acquisitions for the investigated crop fields (summer oat, winter triticale, 
winter barley, winter rye and winter wheat). The range of AOI over the fields varies between $23.4^{\circ}$ and $58.6^{\circ}$. In addition the mean in situ soil moisture and the mean in situ vegetation height are presented, reporting a dry weather period, which led to a soil moisture level of about $20 \mathrm{vol} . \%$ on almost all test fields. In addition the vegetation height is low with a maximum of $24 \mathrm{~cm}$, except for the summer oat field, which is almost bare.

Tab. 1. Incidence angle of the different acquisitions (master: AOI-m, opposite: AOI-o, perpendicular: AOI-p) and mean in situ soil moisture (Mv) and mean in situ vegetation height (Height) for the investigated fields (WT=winter triticale, $\mathrm{WB}=$ winter barley, $\mathrm{WR}=$ winter rye, $\mathrm{WW}=$ winter wheat, $\mathrm{SO}=$ summer oat, $-=$ out of scene)

\begin{tabular}{cccccc}
\hline Fields & $\begin{array}{c}\text { AOI-m } \\
{\left[{ }^{\circ}\right]}\end{array}$ & $\begin{array}{c}\text { AOI-o } \\
{\left[{ }^{\circ}\right]}\end{array}$ & $\begin{array}{c}\text { AOI-p } \\
{\left[{ }^{\circ}\right]}\end{array}$ & $\begin{array}{c}\text { Mv } \\
{[\text { vol. } \%]}\end{array}$ & $\begin{array}{c}\text { Height } \\
{[\mathrm{cm}]}\end{array}$ \\
\hline WT & 49.9 & 39.8 & 53.5 & 19.4 & 23.0 \\
WB & 38.4 & 49.8 & 43.4 & 11.8 & 24.4 \\
WR & 33.9 & 52.9 & 35.2 & 19.9 & 17.4 \\
WW & 58.6 & 26.6 & 55.5 & 16.9 & 14.0 \\
SO & 23.4 & 58.2 & - & 22.7 & 6.75 \\
\hline
\end{tabular}

In Fig. 3a the land use map displays the agricultural, the forested (upper right corner of the scene) and the urban areas (located along the rivers). The incidence angle in Fig. $3 \mathrm{~b}$ illustrates the topography variation dominated by the river systems running predominantly from South to North through the scene, where the steep slopes located on the left edge of the incidence angle image belong to another river system not covered by the scene.

In Fig. 3c-e three out of seven possible results for the mv estimation with the incidence angle constellation master (Fig. 3c), master-opposite (Fig. 3d) and master-oppositeperpendicular (Fig. 3e) are presented exemplarily. The soil moisture values range from 0 to $50 \mathrm{vol} . \%$, whereas white areas represent invalid values, e.g. forest areas. Focusing on the agricultural fields in Fig. 3c the soil moisture is derived with only one acquisition (master) leading to non-invertible areas on the fields with steep incidence angles $\left(<20^{\circ}\right)$ located near the river beds represented by the dark blue regions of Fig. 3b (cf. [2]). In contrast Fig. 3d does not exhibit these non-invertible areas for the overlapping zone of the two acquisitions. Due to the incorporation of a second acquisition for mv inversion with a bi-angular observation the amount of invertible values increases in regions with steep incidence angles and more pixels can be inverted. In Fig. $3 e$ the mv estimation using all three acquisitions in a tri-angular approach improves compared with Fig. 3d mainly in the area where both acquisitions (master, perpendicular) were recorded with steep incidence angles. In Tab. 2 the inversion rates results using seven different incidence angle constellations are summarized for an overlapping area (red frame in Fig. 3) estimated over the agricultural fields. A significant increase of inversion rate is obtained, as two or three acquisitions were included. For a single acquisition an inversion rate of $30-49 \%$ can be achieved, whereas for two acquisitions the rate rises to $55-63 \%$. Finally with the maximum information content of three acquisitions an inversion rate of $71 \%$ is obtained.

Tab. 2. Inversion rate for different incidence angle constellations $(\mathrm{m}=$ master, $\mathrm{o}=$ opposite, $\mathrm{p}=$ perpendicular $)$ on agricultural fields (forest, water and urban areas are not considered) within the red frame (see Fig. 3)

\begin{tabular}{cccc}
\hline One acquisition & master & opposite & perpendicular \\
& $40.32 \%$ & $29.87 \%$ & $48.53 \%$ \\
\hline Two acquisitions & $\mathrm{m}-\mathrm{o}$ & $\mathrm{m}-\mathrm{p}$ & $\mathrm{o}-\mathrm{p}$ \\
& $55.87 \%$ & $63.39 \%$ & $60.71 \%$ \\
\hline Three acquisitions & \multicolumn{3}{c}{$\mathrm{m}-\mathrm{o}-\mathrm{p}$} \\
& & $70.89 \%$ & \\
\hline
\end{tabular}

\subsection{Validation}

In order to investigate the performance of the mv retrieval using seven different incidence angle constellations, a validation was carried out, which compares soil moisture values averaged from three ground measurement points at $0-5 \mathrm{~cm}$ depth with the estimated soil moisture values from the seven different retrieval methods in vol.\%.

For the retrieval of the estimated soil moistures a $13 \times 13$ box around the sampling points leading to 169 looks was taken for comparison considering that only boxes with at least $30 \%$ invertible pixels were included to avoid the influence of non-representative outliers. For the calculation of the RMSE a minimum of five values are taken. Therefore only a few fields are represented in Fig. 4 for the single acquisitions (master, opposite, perpendicular).

Comparing the retrieved soil moisture under vegetation cover for the seven incidence angle constellations the mean level of RMSE incorporating all investigated fields is approximately $7 \mathrm{vol} . \%$, which confirms the stability of the approach for the different crop types and the different retrieval methods. In particular the results of the winter rye field indicate the highest stability with a RMSE below $6.5 \mathrm{vol} . \%$ for all retrieval methods and all incidence angles $\left(33.9^{\circ}, 35.2^{\circ}, 52.9^{\circ}\right)$. Whereas the winter triticale and the winter wheat fields show the highest variability with an RMSE of around $9 \mathrm{vol} . \%$, if retrieval methods with the perpendicular acquisition (perpendicular, oppositeperpendicular) are used. For these constellations the inverted soil moisture from the bare soil component of these fields is overestimated, which might be due to a vegetation layer of $14-23 \mathrm{~cm}$, plus a high incidence angle $\left(>50^{\circ}\right)$ and a roughness component estimated under these vegetation and incidence angle conditions.

The summer oat field shows a RMSE higher than 7vol.\% due to an underestimation of $\mathrm{mv}$ for the master acquisition, which is caused by an erroneous subtraction of the volume layer due to a too small incidence angle $\left(23.4^{\circ}\right)$ for a correct inversion. 


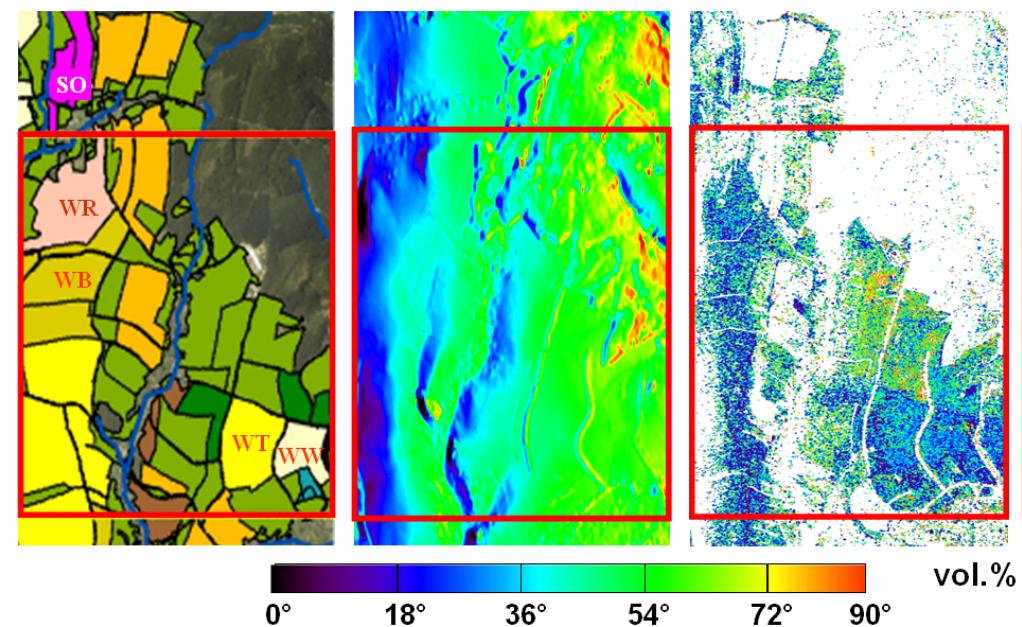

(a)

(b)

(c)

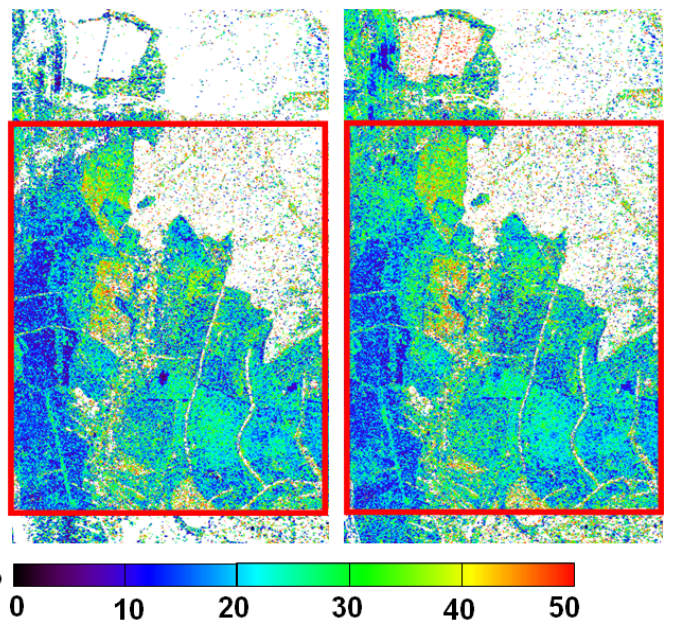

(d)

(e)

Fig. 3. (a) The land use (labels of the test fields are explained in Tab.1), (b) the local incidence angle in degrees as well as (c-e) the estimated soil moisture in vol.\% (averaging window: $4 \mathrm{x} 4)$ is displayed exemplarily for three different incidence angle constellations (3c: master [one acquisition], 3d: master-perpendicular [two acquisitions], 3e: master-opposite-perpendicular [three acquisitions]). White color represents non-invertible pixels; red frame indicates the overlapping zone of the three acquisitions (master, opposite, perpendicular).

Finally comparing all seven retrieval methods the triangular method incorporates the maximum information content and presents the most stable and complete mv inversion for all crop fields.

\section{CONCLUSION}

A modified three component polarimetric decomposition technique incorporating multi-angular acquisitions for mv inversion was developed and applied on fullypolarimetric L-band data acquired by DLR's airborne ESAR sensor in the frame of the OPAQUE campaign in May 2008. The inversion rate increases from 30-49\% for the single, over $55-63 \%$ for the two to $71 \%$ for the three acquisition cases. In addition a validation with ground measurements was conducted for five test fields with different crop types. The analysis of the RMSE for mv retrieval with the seven different incidence angle constellations and including all test fields shows a stable inversion for the majority of methods resulting in a error level of approximately $7 \mathrm{vol} . \%$ with a slight preference for the tri-angular approach incorporating three acquisitions (master-opposite-perpendicular). Finally the results demonstrate how topographic effects in the mv inversion can be compensated, if more than a single angle acquisition is used.

\section{ACKNOWLEDGEMENTS}

The authors would like to thank the OPAQUE team (University of Potsdam, University of Stuttgart and German Research Center for Geoscience (GFZ)) for their contributions to the measurement campaign and $\mathrm{BMBF}$ for supporting the activities.

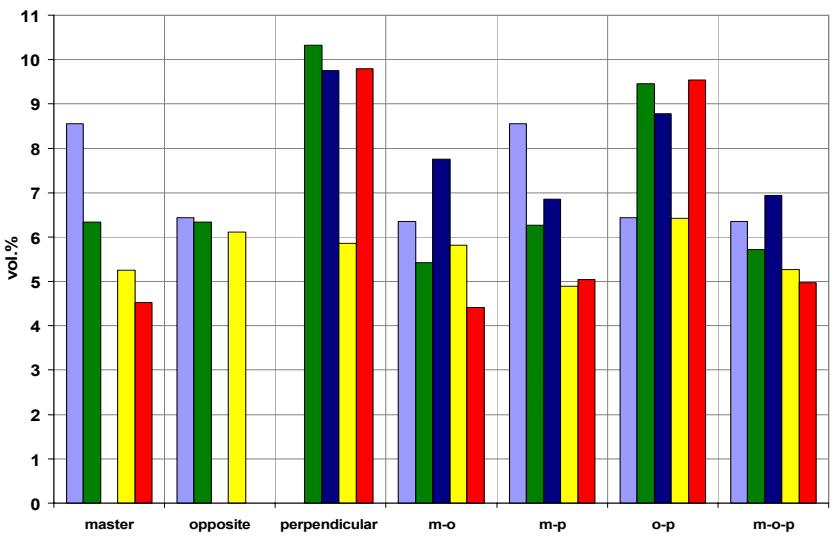

Fig. 4. RMSE of mv estimation in vol. \% for summer oat (bright blue), winter triticale (green), winter barley (dark blue), winter rye (yellow) and winter wheat (red) with the seven different incidence angle constellations $(\mathrm{m}=$ master, $\mathrm{o}=$ opposite, $\mathrm{p}=$ perpendicular)

\section{REFERENCES}

[1] I. Hajnsek, T. Jagdhuber, H Schön \& K.P. Papathanassiou, "Potential of Estimating Soil Moisture under Vegetation Cover by means of PolSAR", IEEE Trans. Geosci. Remote Sensing, 47(2), pp. 442-454, 2009.

[2] T. Jagdhuber, H. Schön, I. Hajnsek, K.P. Papathanassiou, "Soil Moisture Estimation under Vegetation applying Polarimetric Decomposition Techniques", Proc. of the $4^{\text {th }}$ International Workshop on Science and Applications of SAR Polarimetry and Polarimetric Interferometry, Frascati, Italy, 2009.

[3] F. Mattia, T. Le Toan, J.-C., Souyris, G. De Carolis, N. Floury, F. Posa \& G. Pasquariello, "The Effect of Surface Roughness on Multifrequency Polarimetric SAR Data", IEEE Trans. Geosci. Remote Sensing, 35(4), pp. 954-966, 1997.

[4] G.C. Topp, J.L. Davis \& A.P. Annan, "Electromagnetic Determination of Soil Water Content: Measurements in Coaxial Transmission Lines", Water Resources Res., 16(3), pp. 574-582, 1980. 